\title{
Monoclinic elastic and piezoelectric properties of Rochelle salt. Description within the modified Mitsui model
}

\author{
R.R.Levitskii ${ }^{1}$, I.R.Zachek ${ }^{2}$, A.P.Moina ${ }^{1}$ \\ 1 Institute for Condensed Matter Physics \\ of the National Academy of Sciences of Ukraine, \\ 1 Svientsitskii Str., 79011 Lviv, Ukraine \\ 2 Lviv Polytechnic National University, \\ 12 Bandery Street, 79013, Lviv, Ukraine
}

Received August 3, 2005, in final form October 24, 2005

We propose a modification of the two-sublattice Mitsui model that takes into account a coupling with diagonal components of the strain tensor. Within the developed model, we calculate the piezoelectric and elastic characteristics of Rochelle salt related to those strains. In the approximation of zero thermal expansion, we find a set of the model parameters providing a satisfactory agreement with the available experimental data for the elastic constants $c_{i j}^{\mathrm{E}}$ and $c_{i 4}^{\mathrm{E}}$ and for the piezoelectric constants $d_{1 j}$ and $g_{1 j}$.

Key words: Rochelle salt, electrostriction, piezoeffect, elastic constant

PACS: $77.80 . \mathrm{Bh}, 77.22 . \mathrm{Ch}$

\section{Introduction}

Though the crystals of Rochelle salt are the first known ferroelectrics, they keep attracting the physicists due to the curious character of their ferroelectric behavior. For their theoretical description, a two-sublattice Ising model with an asymmetric double-well potential (Mitsui model [1]) is traditionally used. Recently [2,3] we have presented a modification of the conventional Mitsui model, taking into account the piezoelectric coupling with the shear strain $\varepsilon_{4}$. This strain, spontaneous in the ferroelectric phase, can be induced by the electric field $E_{1}$ applied along the axis of spontaneous polarization or by the shear stress $\sigma_{4}$. A good description of the static and dynamic dielectric, piezoelectric, and elastic characteristics of Rochelle salt crystals related to strain $\varepsilon_{4}$ and field $E_{1}$ was obtained $[2,3]$.

The Mitsui model is essentially one-dimensional [4]. It takes into account only the longitudinal (along the axis of spontaneous polarization) components of the ordering dipoles and is incapable of describing the transverse properties of Rochelle 
salt. Recently a 3D version of the Mitsui model was proposed [4,5] - a four-sublattice model that takes into account the space orientation of the dipoles. The transverse properties of Rochelle salt and the effects related to the fields $E_{2}$ and $E_{3}$ were explored.

For the longitudinal properties, however, the new 3D model yields the results identical to those of the two-sublattice model. Therefore, if we restrict ourselves to the effects related to the field $E_{1}$ and to the shear strain $\varepsilon_{4}$ and do not consider the transverse properties, we are free to use the simpler two-sublattice Mitsui model.

In the paraelectric phases the Rochelle salt crystals are orthorhombic, but in the ferroelectric phase the symmetry lowers down to the monoclinic one (space group $P 2{ }_{1} 11$ ). New non-zero components of the elastic and piezoelectric constant tensors arise. In particular, the new monoclinic quantities are the piezomoduli $d_{1 i}\left(g_{1 i}\right)$ and the elastic constants $c_{i 4}^{\mathrm{E}}(i=1,2,3)$ related to the field $E_{1}$ and strain $\varepsilon_{4}$. They are linear functions of the order parameter.

These monoclinic characteristics couple the field $E_{1}$ and strain $\varepsilon_{4}$ to the diagonal components of the strain tensor $\varepsilon_{i}$. The diagonal strains are always present due to the thermal expansion of the crystals. They can also be induced by external stresses, which do now change the crystal symmetry, as well as by the longitudinal electric field $E_{1}$ or shear stress $\sigma_{4}$ via the electrostriction effect. Actually, the monoclinic piezoeffect itself is electrostriction linearized over polarization $P_{1}$ : the constants $g_{1 i}$ give rise to new terms of the $g_{1 i} \varepsilon_{i} P_{1}$ type in the thermodynamic potential. Since $g_{1 i} \sim P_{1}$, they are equivalent to $q_{1 i} \varepsilon_{i} P_{1}^{2}$, where $q_{1 i}$ are the electrostriction constants.

In this work we present another modification of the two-sublattice Mitsui model, including the coupling with the diagonal strains, and calculate the piezoelectric and elastic characteristics of Rochelle salt related to these strains. The proposed modification also permits to describe the effect of hydrostatic pressure or of uniaxial stresses, which do now change the crystal symmetry, on the properties of Rochelle salt. This, however, will be discussed in detail elsewhere.

\section{System thermodynamics in the presence of diagonal strains}

Thermodynamic potential of the piezoelectric Mitsui model without the diagonal strains was obtained within the mean field approximation in the following form [2]:

$$
g=U_{\text {seed }}^{0}+\frac{J+K}{4} \xi^{2}+\frac{J-K}{4} \sigma^{2}-\frac{2 \ln 2}{\beta}-\frac{1}{\beta} \ln \cosh \frac{\gamma+\delta}{2} \cosh \frac{\gamma-\delta}{2},
$$

where

$$
\gamma=\beta\left(\frac{J+K}{2} \xi-2 \psi_{4} \varepsilon_{4}+\mu_{1} E_{1}\right), \quad \delta=\beta\left(\frac{J-K}{2} \sigma+\Delta\right),
$$

$J, K$ are the Fourier-transforms (at $\mathbf{k}=0$ ) of the constants of interaction between pseudospins belonging to the same and to different sublattices, respectively. The parameter $\Delta$ describes the asymmetry of the double well potential; $\mu_{1}$ is the effective 
dipole moment. The model parameter $\psi_{4}$ describes the internal field created by the piezoelectric coupling with the shear strain $\varepsilon_{4}$.

$$
U_{\text {seed }}^{0}=\frac{v}{2} c_{44}^{E 0} \varepsilon_{4}^{2}-v e_{14}^{0} \varepsilon_{4} E_{1}-\frac{v}{2} \chi_{11}^{\varepsilon 0} E_{1}^{2}
$$

is a "seed" energy of the crystal lattice which forms the asymmetric double-well potential for the pseudospins. Here $v$ is the unit cell volume of the model, twice smaller than the actual unit cell volume of a Rochelle salt crystal. In order to find the corresponding susceptibilities, elastic constants, etc., we assume that a longitudinal electric field $E_{1}$ and a shear stress $\sigma_{4}$ are applied.

Parameters of ferro- and antiferroelectric ordering $\xi$ and $\sigma$ are determined from the saddle point of the thermodynamic potential (1): a minimum of $g$ with respect to $\xi$ and a maximum with respect to $\sigma$ are realized at equilibrium. The corresponding equations are [2]

$$
\xi=\frac{\sinh \gamma}{\cosh \gamma+\cosh \delta}, \quad \sigma=\frac{\sinh \delta}{\cosh \gamma+\cosh \delta} .
$$

The diagonal strains will be taken into account in a linear approximation. Similarly to the method, used in [6] for description of hydrostatic pressure effects in $\mathrm{H}$-bonded crystals of the $\mathrm{KD}_{2} \mathrm{PO}_{4}$ type, we simply assume that the interaction constants as well as the asymmetry parameter are linear functions of the strains:

$$
J=J_{0}+\sum_{i=1}^{3} \psi_{1 i} \varepsilon_{i}, K=K_{0}+\sum_{i=1}^{3} \psi_{2 i} \varepsilon_{i}, \Delta=\Delta_{0}+\sum_{i=1}^{3} \psi_{3 i} \varepsilon_{i}
$$

Diagonal strains, arising due to the thermal expansion or induced by external stresses that do not affect the crystal symmetry, should not change the form or symmetry of the model Hamiltonian. Neither do they change the form of the pseudospin part of the thermodynamic potential, except that the parameters $J, K, \Delta$ should be renormalized according to (3). Let us note that the parameters of this renormalization enter the thermodynamic potential only as the linear combinations

$$
\psi_{i}^{+}=\frac{1}{2}\left(\psi_{1 i}+\psi_{2 i}\right), \quad \psi_{i}^{-}=\frac{1}{2}\left(\psi_{1 i}-\psi_{2 i}\right), \quad \psi_{3 i} .
$$

The phenomenological part of the potential, which is independent (explicitly) of the spin variables, should be modified as

$$
U_{\text {seed }}^{0} \rightarrow U_{\text {seed }}=v \sum_{i j=1}^{3} c_{i j}^{0} \varepsilon_{i} \varepsilon_{j}+v c_{44}^{E 0} \varepsilon_{4}^{2}-v e_{14}^{0} \varepsilon_{4} E_{1}-\frac{v}{2} \chi_{11}^{\varepsilon 0} E_{1}^{2} .
$$

It now contains new terms related to the diagonal strains. Here $c_{i j}^{0}$ are the "seed" elastic constants describing the phenomenological contribution of the crystal lattice into the observed elastic constants $c_{i j}$. The "seed" temperature independent contributions to the piezoelectric constants $e_{1 i}$ or elastic constants $c_{i 4}(i=1,2,3)$ should be equal to zero, since both $e_{1 i}$ and $c_{i 4}$ are zeros outside the ferroelectric phase. 
Thus, using the thermodynamic potential (1), with $J, K, \Delta$ changed according to (3) and with $U_{\text {seed }}^{0} \rightarrow U_{\text {seed }}$, from the following thermodynamic relations

$$
\frac{1}{v}\left(\frac{\partial g}{\partial \varepsilon_{i}}\right)_{E 1}=\sigma_{i}, \quad-\frac{1}{v}\left(\frac{\partial g}{\partial E_{1}}\right)_{\sigma}=P_{1}, \quad i=1,2,3,4,
$$

we obtain the equations for strains and polarization

$$
\begin{aligned}
\sigma_{i} & =\sum_{j=1}^{3} c_{i j}^{0} \varepsilon_{j}-\frac{1}{2 v} \psi_{i}^{+} \xi^{2}+\frac{1}{2 v} \psi_{i}^{-} \sigma^{2}-\frac{1}{v} \psi_{3 i} \sigma, \quad i=1,2,3, \\
\sigma_{4} & =c_{44}^{E 0} \varepsilon_{4}-e_{14}^{0} E_{1}+2 \frac{\psi_{4}}{v} \xi \\
P_{1} & =e_{14}^{0} \varepsilon_{4}+\chi_{11}^{\varepsilon 0} E_{1}+\frac{\mu_{1}}{v} \xi .
\end{aligned}
$$

Here $\sigma_{i}$ at $i=1,2,3$ are the diagonal components of the stress tensor $\left(\sigma_{1}=\sigma_{2}=\right.$ $\sigma_{3}=-p$ for hydrostatic pressure). Remember that we consider only the stresses that do not affect the crystal symmetry as well as the shear stress $\sigma_{4}$ which in the paraelectric phases lowers the symmetry down to the monoclinic one.

As one can see, even at $\sigma_{i}=0$ the diagonal strains $\varepsilon_{i}$, which in this case describe the thermal expansion of the crystals, are always different from zero.

\section{Physical characteristics of Rochelle salt related to diagonal strains}

From equations (4)-(6) we can derive the elastic and piezoelectric characteristics of Rochelle salt related to diagonal strains:

- coefficients of piezoelectric stress $(i=1,2,3)$

$$
e_{1 i}=\frac{\beta \mu_{1}}{2 v} \frac{\varphi_{4 i}}{\varphi_{2}}
$$

- elastic constants at constant electric field $(i, j=1,2,3)$

$$
\begin{aligned}
c_{i j}^{\mathrm{E}}= & c_{i j}^{E 0}-\frac{\beta}{2 v \varphi_{2}}\left\{\left[\psi_{i}^{+} \psi_{j}^{+} \xi^{2}+\left(\psi_{i}^{-} \sigma-\psi_{3 i}\right)\left(\psi_{j}^{-} \sigma-\psi_{3 j}\right)\right] \lambda_{1}\right. \\
& +\left[\psi_{i}^{+}\left(\psi_{j}^{-} \sigma-\psi_{3 j}\right)+\psi_{j}^{+}\left(\psi_{i}^{-} \sigma-\psi_{3 i}\right)\right] \xi \lambda_{2}+\frac{\beta}{4}\left[(K-J) \psi_{i}^{+} \psi_{j}^{+} \xi^{2}\right. \\
& \left.\left.-(K+J)\left(\psi_{i}^{-} \sigma-\psi_{3 i}\right)\left(\psi_{j}^{-} \sigma-\psi_{3 j}\right)\right]\left(\lambda_{1}^{2}-\lambda_{1}^{2}\right)\right\}, \\
c_{i 4}^{\mathrm{E}}= & \frac{\beta \psi_{4}}{v} \frac{\varphi_{4 i}}{\varphi_{2}}
\end{aligned}
$$

where

$$
\begin{aligned}
\varphi_{4 i} & =\psi_{i}^{+} \xi \varphi_{3}+\left(\psi_{i}^{-} \sigma-\psi_{3 i}\right) \lambda_{2}, \\
\varphi_{2} & =1-\frac{\beta J}{2} \lambda_{1}-\beta^{2} \frac{K^{2}-J^{2}}{16}\left(\lambda_{1}^{2}-\lambda_{2}^{2}\right), \quad \varphi_{3}=\lambda_{1}+\frac{\beta(K-J)}{4}\left(\lambda_{1}^{2}-\lambda_{2}^{2}\right), \\
\lambda_{1} & =1-\xi^{2}-\sigma^{2}, \quad \lambda_{2}=2 \xi \sigma .
\end{aligned}
$$


The piezoelectric constants $d_{1 i}, h_{1 i}$, and $g_{1 i}(i=1,2,3)$ as well as the elastic constants $c_{i j}^{P}$ at constant polarization read

$$
d_{1 i}=\sum_{j=1}^{4} s_{i j}^{\mathrm{E}} e_{1 j}, \quad h_{1 i}=\frac{e_{1 j}}{\chi_{11}^{\varepsilon}}, \quad g_{1 i}=\frac{d_{1 j}}{\chi_{11}^{\sigma}}, \quad c_{i j}^{P}=c_{i j}^{\mathrm{E}}+e_{1 i} h_{1 j} .
$$

where $s_{i j}^{\mathrm{E}}$ is the matrix of elastic compliances, inverse to the matrix of elastic constants $c_{i j}^{\mathrm{E}}$, whereas

$$
\chi_{11}^{\sigma}=\chi_{11}^{\varepsilon}+\sum_{i=1}^{4} e_{1 i} d_{1 i}
$$

is the dielectric susceptibility of a free crystal. In the paraelectric phases and at zero diagonal strains it formally coincides with the found earlier [3] expression

$$
\chi_{11}^{\sigma}(0)=\chi_{11}^{\sigma 0}+\frac{\beta\left(\mu_{1}^{\prime}\right)^{2}}{2 v} \frac{\varphi_{3}}{\varphi_{2}-\Lambda \varphi_{3}},
$$

where

$$
\Lambda=\frac{2 \beta \psi_{4}^{2}}{v c_{44}^{E 0}}, \quad \mu_{1}^{\prime}=\mu_{1}-2 \psi_{4} d_{14}^{0}, \quad d_{14}^{0}=\frac{e_{14}^{0}}{c_{44}^{E 0}}, \quad \chi_{11}^{\sigma 0}=\chi_{11}^{\varepsilon 0}+e_{14}^{0} d_{14}^{0},
$$

and

$$
\chi_{11}^{\varepsilon}=\chi_{11}^{\varepsilon 0}+\frac{\beta \mu_{1}^{2}}{2 v} \frac{\varphi_{3}}{\varphi_{2}}
$$

is the dielectric susceptibility of a clamped crystal.

As one can easily verify, the quantities $e_{1 i}, d_{1 i}, h_{1 i}, g_{1 i}, c_{i 4}^{\mathrm{E}}, c_{i 4}^{P}(i=1,2,3)$ differ from zero only at non-zero polarization, in agreement with the symmetry considerations.

From (11) and using (2) we obtain an equation for the transition temperatures

$$
\cosh ^{2}\left[\beta_{\mathrm{C}}\left(\frac{\Delta}{2}-\frac{K-J}{4} \sigma\right)\right]=\beta_{\mathrm{C}}\left[\frac{K+J}{4}+\frac{2 \psi_{4}^{2}}{v c_{44}^{E 0}}\right] .
$$

This equation gives the pressure dependences of transition temperatures when the model parameters $J, K, \Delta$ are being renormalized by pressure-induced strains according to (3). Let us remind that in Rochelle salt at $p=0$ there are two transitions $T_{\mathrm{C} 1}=255 \mathrm{~K}$ and $T_{\mathrm{C} 2}=297 \mathrm{~K}$.

\section{Numerical analysis}

Thermal diagonal strains, determined from (4), can essentially affect the shape of the phase diagram of the Mitsui model. In the present work we shall make a further approximation, neglecting the diagonal strains induced by thermal expansion in the absence of external pressures, i.e. we put $\varepsilon_{i}=0(i=1,2,3)$ at zero pressure. The quantities $J_{0}, K_{0}, \Delta_{0}$ are then chosen equal to the values of $J, K, \Delta$, used within 
the earlier model [2]. The rest of the parameters occurring both in the former and in the present models $\left(\mu_{1}, c_{44}^{E 0}, d_{14}^{0}, \chi_{11}^{\sigma 0}\right)$ remain unchanged.

We need to determine nine parameters $\psi_{i}^{ \pm}, \psi_{3 i}$. The fitting procedure was done as follows.

Firstly, three parameters, namely $\psi_{2}^{ \pm}, \psi_{32}$, can be expressed via the other six using the experimental values of the sum $c_{24}^{\mathrm{E}}+c_{34}^{\mathrm{E}}[7]$ at three temperatures in the ferroelectric phase.

Secondly, we demand that the two transition temperatures calculated with (13) at hydrostatic pressure of $1 \mathrm{kbar}$ should be in agreement with the experimental data of $[8,9]$, i.e. the theory should give $\mathrm{d} T_{\mathrm{C} 1} / \mathrm{d} p=3.8 \mathrm{~K} / \mathrm{kbar}$ and $\mathrm{d} T_{\mathrm{C} 2} / \mathrm{d} p=11 \mathrm{~K} / \mathrm{kbar}$. Thus, two of the remaining six parameters, say $\psi_{1}^{-}$and $\psi_{31}$, may also be expressed via the four others.

Thirdly, the temperature dependences of the elastic constants $c_{i j}^{\mathrm{E}}(i=1,2,3)$ are very weak [7]; therefore, the last four free parameters should minimize the contribution of the pseudospin subsystem into the temperature variation of $c_{i j}^{\mathrm{E}}$. For the same reason, the "seed" elastic constants $c_{i j}^{0},(i=1,2,3)$ are taken temperature independent and close to the experimental values of $c_{i j}^{\mathrm{E}}[7]$.

Finally, varying the remaining free parameters, we tried to fit the theoretical curves to the data for $d_{11}(T)$ [10] and $g_{1 i}(T)$ [11].

The adopted values are given in Table 1 . The nine new model parameters $\psi_{i}^{ \pm}$, $\psi_{3 i}$ provide a temperature behavior of nine elastic constants $c_{i j}^{\mathrm{E}}$ and $c_{i 4}^{\mathrm{E}}$, as well as of six piezoelectric constants $d_{1 j}$ and $g_{1 j}$ compliant with the experiment $[7,10,11]$. The results of calculations are presented in figure 1.

Table 1. Theory parameters.

\begin{tabular}{ccccccc}
\hline \hline$J_{0} / k_{\mathrm{B}}$ & $\begin{array}{c}K_{0} / k_{\mathrm{B}} \\
\mathrm{K}\end{array}$ & $\Delta_{0} / k_{\mathrm{B}}$ & $\psi_{4} / k_{\mathrm{B}}$ & $\begin{array}{c}c_{44}^{E 0} \\
\mathrm{dyn} / \mathrm{cm}^{2}\end{array}$ & $\begin{array}{c}d_{14}^{0} \\
\mathrm{esu} / \mathrm{dyn}\end{array}$ & $\chi_{11}^{\sigma 0}$ \\
\hline 797.36 & 1468.83 & 737.33 & -760 & $12.8 \cdot 10^{10}$ & $1.9 \cdot 10^{-8}$ & 0.363 \\
\hline \hline
\end{tabular}

\begin{tabular}{ccccccccc}
\hline \hline$\psi_{1}^{+} / k_{\mathrm{B}}$ & $\psi_{2}^{+} / k_{\mathrm{B}}$ & $\psi_{3}^{+} / k_{\mathrm{B}}$ & $\psi_{1}^{-} / k_{\mathrm{B}}$ & $\begin{array}{c}\psi_{2}^{-} / k_{\mathrm{B}} \\
\mathrm{K}\end{array}$ & $\psi_{3}^{-} / k_{\mathrm{B}}$ & $\psi_{31} / k_{\mathrm{B}}$ & $\psi_{32} / k_{\mathrm{B}}$ & $\psi_{33} / k_{\mathrm{B}}$ \\
\hline-13500 & 5965 & 6000 & -12266 & 25088 & 15890 & -13625 & 19357 & 13200 \\
\hline \hline
\end{tabular}

\begin{tabular}{cccccc}
\hline \hline$c_{11}^{0}$ & $c_{12}^{0}$ & $c_{13}^{0}$ & $c_{22}^{0}$ & $c_{23}^{0}$ & $c_{33}^{0}$ \\
\multicolumn{5}{c}{$10^{11} \mathrm{dyn} / \mathrm{cm}^{2}$} \\
\hline 2.80 & 1.74 & 1.49 & 4.14 & 1.97 & 3.94 \\
\hline \hline
\end{tabular}

$v=0.5219[1+0.00013(T-190)] \cdot 10^{-21} \mathrm{~cm}^{3}, \quad \mu_{1}=[2.52+0.0066(297-T)] \cdot 10^{-18} \mathrm{esu} \mathrm{cm}$.

The obtained description of experimental data appears to be satisfactory. The elastic constants at constant polarization $\left|c_{24}^{P}+c_{34}^{P}\right|$ are essentially lower than $\mid c_{24}^{\mathrm{E}}+$ $c_{34}^{\mathrm{E}} \mid$ (negligibly small in the scale chosen in figure 1), which agrees with the experiment. No perceptible difference between $c_{i j}^{P}$ and $c_{i j}^{\mathrm{E}}(i, j=1,3,3)$ was detected. 
Temperature behavior of the piezoelectric constants $e_{1 j}$ and elastic constants $c_{i 4}^{\mathrm{E}}$ coincides within a constant factor $\mu_{1} / 2 \psi_{4}$ (see (7), (9)). The piezoelectric constants $d_{1 j}$ have peaks at the Curie temperatures and jump to zero at the transitions to the paraelectric phases. The temperature curves of the piezoelectric constants $g_{1 j}$ are qualitatively similar to that of spontaneous polarization.

It should be also mentioned that there is also some recent data of X-ray multiple diffraction $[13,14]$ for the piezoelectric constants $d_{1 i}$ at $295 \mathrm{~K}$, not included in our fitting. The reasons for this are as follows. The values of $d_{1 i}$ obtained there are in contradiction with the data of $[10,11]$, exceeding the latter by several times. We had to choose the set of experimental data on which we would base our calculations. The recent data would, of course, be preferable. It is difficult, however, to do the fitting, using only a single experimental point for each $d_{1 i}$. Therefore, we based our calculations on the old but full temperature curves of $d_{1 i}$ and $g_{1 i}$ of $[10,11]$. Nevertheless, it is of a very high interest to have data for the temperature dependences of the monoclinic piezoelectric constants of Rochelle salt measured by modern methods and spanning the entire ferroelectric phase.

Our further studies will concern the thermal expansion and hydrostatic pressure effect on the Rochelle salt crystals. The values of the model parameters will, of course, change. More recent data for the temperature dependence of the piezoelectric constants should be also taken into account, if available.

The fitting procedure in the case of non-zero thermal strains is rather nontrivial. It requires a special exploration of the phase diagram of the Mitsui model with the temperature dependent interaction parameters. The main difficulty is that the region of the phase diagram (drawn in the interaction parameters coordinates) where the system undergoes two second order phase transitions is rather narrow. Perceptible changes of the model parameters with temperature (due to thermal strains) or pressure can draw the system out of this region. It would imply, for instance, that Rochelle salt undergoes other phase transitions, in addition to those at 255 and $297 \mathrm{~K}$. Though there are some data indicating that the third transition indeed occurs near $212 \mathrm{~K}$ (see [12]), its existence is strongly questionable. No additional phase transition in Rochelle salt induced by hydrostatic pressure has been observed either.

\section{Concluding remarks}

We proposed a generalization of the Mitsui model, which along with the piezoelectric coupling with the shear strain $\varepsilon_{4}$ also takes into account the diagonal strains. The developed model is used for description of monoclinic piezoelectric and elastic characteristics of Rochelle salt crystals. Within the mean field approximation we derive equations for polarization and lattice strains. Numerical analysis of the obtained results is performed in the approximation of zero thermal expansion. A set of the model parameters is found, providing a quantitative agreement with the experimental data for the temperature dependences of the elastic constants $c_{i j}^{\mathrm{E}}$ and $c_{i 4}^{\mathrm{E}}$ as well 

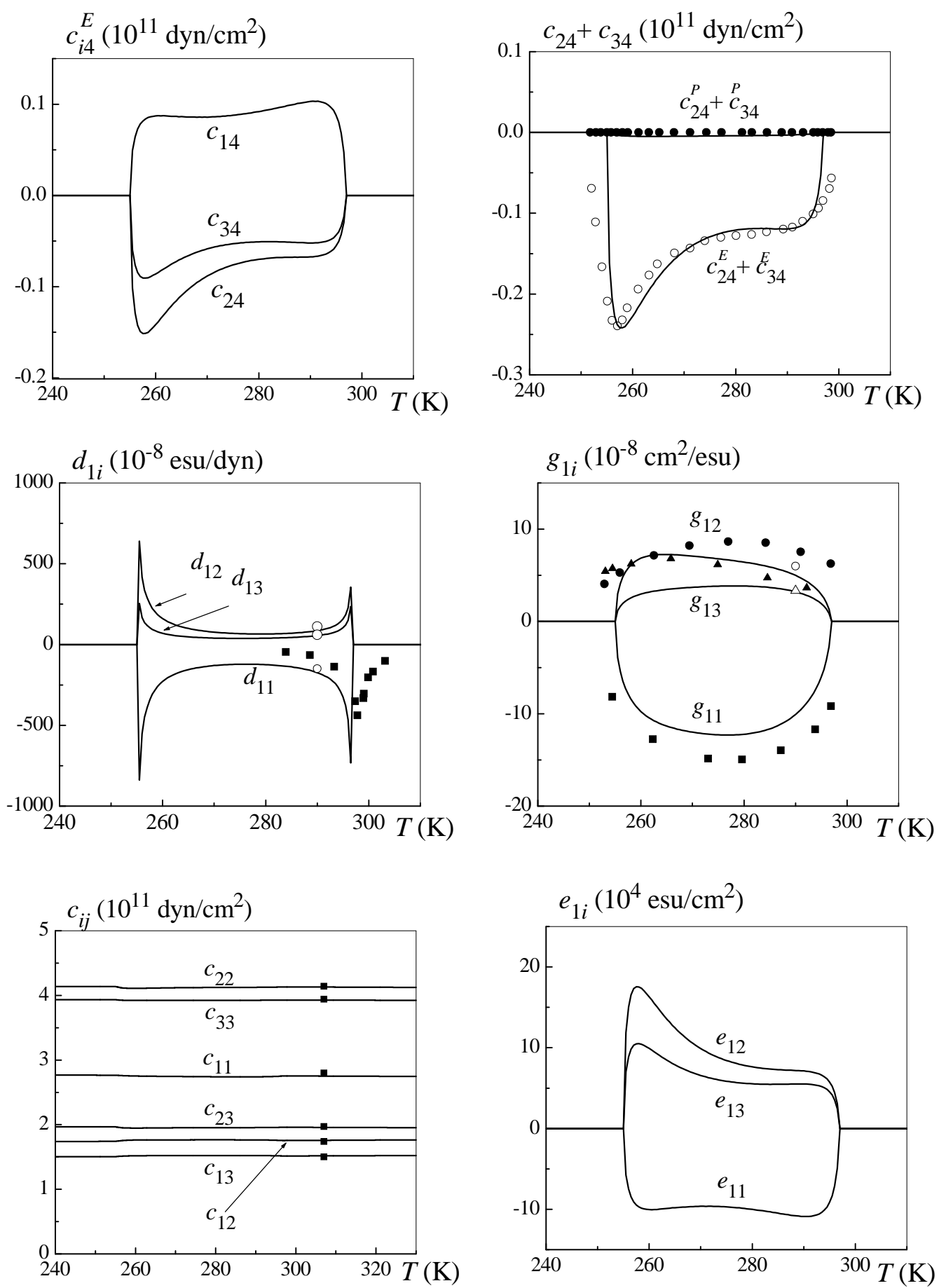

Figure 1. Temperature dependences of elastic and piezoelectric characteristics of Rochelle salt related to the strains $\varepsilon_{i}(i=1,2,3)$. Experimental data are taken from: for $c_{i j}: \mathbf{\square}, \circ, \bullet-[7]$, for $d_{1 j}: \mathbf{\square}-[10], \circ-[11]$, for $g_{1 j}-[11]$ (the data of [11] are obtained at $E_{1}=5 \cdot 10^{5} \mathrm{~V} \mathrm{~m}^{-1}$ or extrapolated to $\left.E_{1}=0\right)$. 
as of the piezoelectric constants $d_{1 j}$ and $g_{1 j}$. Calculations of the hydrostatic pressure effects in Rochelle salt within the proposed model taking into account thermal expansion are now under way.

\section{Acknowledgement}

The authors acknowledge support of Fundamental Research State Fund of Ukraine, project No. 02.07/00310.

\section{References}

1. Mitsui T., Phys. Rev., 1958, 111, 1259.

2. Levitskii R.R., Zachek I.R., Verkholyak T.M., Moina A.P., Phys. Rev. B., 2003, 67, 174112 .

3. Moina A.P., Levitskii R.R., Zachek I.R., Phys. Rev. B, 2005, 71, 134108.

4. Stasyuk I.V, Velychko O.V., Ferroelectrics, 2005, 316, 51.

5. Stasyuk I.V, Levitskii R.R., Zachek I.R., Vdovych A.S., Velychko O.V., to be published.

6. Stasyuk I.V., Levitskii I.V., Moina A.P., Phys. Rev. B., 1999, 59, 8530.

7. Numerical Data and Functional Relationships in Science and Technology, edited by K.-H. Hellwege, A.M. Hellwege. Landolt-Bornstein, New Series. Group III: Crystal and Solid State Physics, vol. 16. Pt. b Springer-Verlag, Berlin, 1982.

8. Bancroft D., Phys. Rev., 1938, 53, 587.

9. Samara G.A., J. Chem. Solids, 1965, 26, 121.

10. Fotchenkov A.A. Kristallografia, 1960, 5, No. 3, 415-420 (in Russian).

11. Schmidt G. Zeitsch. Physik, 1961, 161, 579.

12. Dey P.K., Som K.K., Chowdhury K.R., Sarkar B.K., Chaudhuri B.K., Phys. Rev. B, 1993, 47, 3001.

13. dos Santos A.O. et al, J. Phys.: Condens. Matter, 2001, 13, 104976.

14. dos Santos A.O., Cardoso L.P., Sasaki J.M., Miranda M.A.R., Melo F.E.A., J. Phys.: Condens. Matter, 2003, 15, 7835. 


\title{
Моноклінні п'єзоелектричні і пружні властивості сегнетової солі. Опис в рамках модифікованої моделі Міцуї
}

\author{
Р.Р. Левицький ${ }^{1}$, І.Р.Зачек ${ }^{2}$, А.П. Моїна ${ }^{1}$ \\ 1 Інститут фізики конденсованих систем НАН України, \\ 79011 Львів, вул. Свєнціцького, 1 \\ 2 Національний університет “Львівська політехніка", \\ 79013, м. Львів, вул. С. Бандери, 12, Україна
}

Отримано 3 серпня 2005 р., в остаточному вигляді 24 жовтня $2005 \mathrm{p}$.

Запропоновано модифікацію двопідграткової моделі Міцуї, що враховує взаємодії з діагональними компонентами тензора деформації. В їі рамках розраховано пов'язані з цими деформаціями п'єзоелектричні та пружні характеристики кристалів сегнетової солі. Проведено числовий аналіз отриманих результатів. У наближенні нульових теплових деформацій знайдено набір параметрів теорії, який забезпечує задовільний кількісний опис наявних експериментальних даних для пружних сталих $c_{i j}^{\mathrm{E}}$ і $c_{i 4}^{\mathrm{E}}$ та п'єзомодулів $d_{1 j}$ і $g_{1 j}$.

Ключові слова: сегнетова сіль, електрострикція, п'єзоефект, пружна стала

PACS: $77.80 . \mathrm{Bh}, 77.22 . \mathrm{Ch}$ 